\title{
Seeing More Is Knowing More: V3D Enables Real-Time 3D Visualization and Quantitative Analysis of Large-Scale Biological Image Data Sets
}

\author{
Hanchuan Peng and Fuhui Long \\ Howard Hughes Medical Institute
}

Everyone understands seeing more is knowing more. However, for large-scale 3D microscopic image analysis, it has not been an easy task to efficiently visualize, manipulate and understand high-dimensional data in 3D, 4D or 5D spaces. We developed a new 3D+ image visualization and analysis platform, V3D, to meet this need. The V3D system provides 3D visualization of gigabyte-sized microscopy image stacks in real time on current laptops and desktops. V3D streamlines the online analysis, measurement and proofreading of complicated image patterns by combining ergonomic functions for selecting a location in an image directly in 3D space and for displaying biological measurements, such as from fluorescent probes, using the overlaid surface objects. V3D runs on all major computer platforms and can be enhanced by software plug-ins to address specific biological problems. To demonstrate this extensibility, we built a V3Dbased application, V3D-Neuron, to reconstruct complex 3D neuronal structures from high-resolution brain images. V3D-Neuron can precisely digitize the morphology of a single neuron in a fruitfly brain in minutes, with about a 17 -fold improvement in reliability and tenfold savings in time compared with other neuron reconstruction tools. Using V3D-Neuron, we demonstrate the feasibility of building a high-resolution 3D digital atlas of neurite tracts in the fruitfly brain. V3D can be easily extended using a simple-to-use and comprehensive plugin interface. 